hep-th/0305247

\title{
A Charged Rotating Black Ring
}

\author{
Henriette Elvang \\ Department of Physics, UCSB, Santa Barbara, CA 93106 \\ elvang@physics.ucsb.edu
}

October 8, 2018

\begin{abstract}
We construct a supergravity solution describing a charged rotating black ring with $S^{2} \times S^{1}$ horizon in a five dimensional asymptotically flat spacetime. In the neutral limit the solution is the rotating black ring recently found by Emparan and Reall. We determine the exact value of the lower bound on $J^{2} / M^{3}$, where $J$ is the angular momentum and $M$ the mass; the black ring saturating this bound has maximum entropy for the given mass. The charged black ring is characterized by mass $M$, angular momentum $J$, and electric charge $Q$, and it also carries local fundamental string charge. The electric charge distributed uniformly along the ring helps support the ring against its gravitational self-attraction, so that $J^{2} / M^{3}$ can be made arbitrarily small while $Q / M$ remains finite. The charged black ring has an extremal limit in which the horizon coincides with the singularity.
\end{abstract}




\section{Introduction}

Recently, Emparan and Reall [1] found an exact vacuum solution describing a rotating black ring in a five dimensional asymptotically flat spacetime. The black ring solution is the first explicit example of non-uniqueness in higher dimensional gravity in the sense that the asymptotically determined quantities do not uniquely specify the solution: in five dimensions there exist asymptotically flat vacuum solutions with the same mass and angular momentum, but with distinct horizon topologies - one is the rotating black hole with $S^{3}$ horizon and the other is the rotating black ring with $S^{2} \times S^{1}$ horizon. We extend this non-uniqueness result to charged solutions of low energy heterotic string theory.

In five dimensions, the Myers-Perry black hole [2] is characterized by the mass $M$ and two independent angular momenta, $J_{1}$ and $J_{2}$. Taking $J=J_{1}$ and $J_{2}=0$, the dimensionless ratio constructed from $J$ and $M$ has an upper bound,

$$
\frac{J^{2}}{M^{3}} \leq \frac{32}{27 \pi} \quad \text { (black hole) }
$$

Emparan and Reall showed that for the black ring with mass $M$ and angular momentum $J$ the dimensionless ratio $J^{2} / M^{3}$ has a lower bound,

$$
\frac{J^{2}}{M^{3}} \geq k \frac{32}{27 \pi} \quad \text { (neutral black ring) }
$$

and they found $k \approx 0.8437$ [1]. In this paper we show that the exact value is $k=27 / 32$. The solution with $J^{2} / M^{3}=1 / \pi$ is the black ring that maximizes the

entropy for the given mass. For $\frac{1}{\pi} \leq \frac{J^{2}}{M^{3}} \leq \frac{32}{27 \pi}$ there are spherical black hole solutions and black ring solutions with the same values of $J$ and $M$.

The interpretation of the lower bound on $J^{2} / M^{3}$ is that for a given mass it takes a certain angular momentum to balance the gravitational self-attraction of the ring. An electric charge distributed uniformly around the black ring would help support the ring so that the ratio $J^{2} / M^{3}$ could be made arbitrarily small. We find that this is indeed possible.

Applying the solution generating techniques of Hassan and Sen [3] to the rotating black ring, we find a solution describing a charged rotating black ring. This is a solution of the low energy limit of heterotic string theory (heterotic supergravity) and besides carrying a $\mathrm{U}(1)$ electric charge $Q$, the black ring also carries local fundamental string charge. The charged black ring can be viewed as the field of a rotating excited loop of fundamental string with electric charged added. We find that the ratio $J^{2} / M^{3}$ can be made arbitrarily small, while the dimensionless ratio of charge to mass approaches a constant. The charge and mass satisfy $|Q| \leq M$, independent of the angular momentum. We also compute the magnetic moment and gyromagnetic 
ratio. The charged rotating black ring has an extremal limit for which the ring is extremally charged, $|Q|=M$. For the extremal solution, the horizon coincides with the singularity.

It is unknown if supersymmetric black rings exist. In [4] all supersymmetric solutions of $N=1$ minimal supergravity in five dimensions are constructed. Furthermore, as a first uniqueness result, it is argued in [5] that the only supersymmetric, asymptotically flat black hole solutions in this theory are the Breckenridge, Myers, Peet, and Vafa (BMPV) [6] black holes, which are characterized by their mass and angular momentum. However, we have here a black ring solution not to minimal supergravity, but to heterotic supergravity with five dimensions compactified. Hence the uniqueness result of [5] does not exclude the possibility of a supersymmetric black ring for which the extremal limit of the black ring found in this paper may be a candidate.

The matter of uniqueness is interesting in its own right, but is also important for the string theory calculations of the entropy of supersymmetric or nearly supersymmetric black holes [7]. For these derivations it is assumed that the black hole solutions are specified uniquely by their asymptotic charges.

The solutions presented in this paper are not uniquely specified by their asymptotic charges. For a certain range of parameters there are charged rotating black rings and spherical black holes (obtained by applying the Hassan-Sen transformation to a Myers-Perry black hole with a single nonzero angular momentum) with the same asymptotic charges. The extremal limit of the charged black ring hasvanishing horizon area.

The paper is organized as follows. We consider the neutral rotating black ring in section 2] in section 2.1] we review the neutral black ring of [1] and in section 2.2] we derive the exact lower bound of $J^{2} / M^{3}$ for the black ring. We review the HassanSen solution generating technique in section 3. The Hassan-Sen technique gives the transformed solutions implicitly. Starting from quite general solutions we offer in appendix A.1 and appendix A.2 explicit expressions for the transformed solutions. In section 4, we apply the Hassan-Sen transformation to the rotating black ring to obtain the charged ring solution (section 4.1). We investigate the physical properties (section 4.2) and study an extremal limit of the charged black ring (section 4.3). We compare the local behavior of the charged black ring to the local behavior of a charged black string obtained by applying the Hassan-Sen transformation to a boosted black string (section 4.4). Somewhat unrelated to the black rings we discuss in section [5 solutions for charged black strings and their extremal limits. We summarize and discuss the results in section 6 . 


\section{The Black Ring}

Emparan and Reall found vacuum solutions describing black rings with $S^{2} \times S^{1}$ horizons in a five dimensional asymptotically flat spacetime. The static black ring solution [8] has conical singularities preventing the ring from collapsing, but these conical singularities can be avoided if the ring is rotating fast enough to provide a force to balance the ring under its own gravitational attraction [1]. We review the rotating black ring in section 2.1, and in section 2.2 we derive the exact lower bound on $J^{2} / M^{3}$.

\subsection{Review of the Neutral Rotating Black Ring}

The metric of the black ring was obtained by a Wick rotation of a metric in [9]. The solution is characterized by a parameter $\nu$ and a scaling $A$. Written in C-metric coordinates (we adopt the notation of and follow closely Ref. [1]) the metric is

$$
\begin{aligned}
& d s^{2}=-\frac{F(x)}{F(y)}\left(d t+\sqrt{\frac{\nu}{\xi_{1}}} \frac{\xi_{2}-y}{A} d \psi\right)^{2} \\
&+\frac{1}{A^{2}(x-y)^{2}}\left[-F(x)\left(G(y) d \psi^{2}+\frac{F(y)}{G(y)} d y^{2}\right)\right. \\
&\left.+F(y)^{2}\left(\frac{d x^{2}}{G(x)}+\frac{G(x)}{F(x)} d \phi^{2}\right)\right],
\end{aligned}
$$

where

$$
F(\xi)=1-\xi / \xi_{1}, \quad G(\xi)=1-\xi^{2}+\nu \xi^{3}
$$

It is assumed that $0<\nu<2 /(3 \sqrt{3})$ which guarantees that $G(\xi)$ has three distinct real roots, $\xi_{2}, \xi_{3}$, and $\xi_{4}$. The roots can be ordered as $-1<\xi_{2}<0<1<\xi_{3}<\xi_{4}<\frac{1}{\nu}$.

Analyzing the metric (2.1), one finds that in order to keep the signature Lorentzian and to avoid conical singularities, the coordinate ranges are required to be

$$
\xi_{2} \leq x \leq \xi_{3}, \quad y<\xi_{2}
$$

and the angular coordinates $\psi$ and $\phi$ must have periodicities

$$
\Delta \psi=\Delta \phi=\frac{4 \pi \sqrt{F\left(\xi_{2}\right)}}{G^{\prime}\left(\xi_{2}\right)} .
$$

Furthermore $\xi_{1} \geq \xi_{3}$. If $\xi_{1}=\xi_{3}$ the solution (2.1) describes a black hole with $S^{3}$ horizon topology. A coordinate transformation [1] identifies it as the Myers-Perry rotating black hole [2] in five dimensions with one rotation parameter set to zero. In 
the following we assume $\xi_{1}>\xi_{3}$. Since the orbit of $g_{\phi \phi}$ then vanishes at both $x=\xi_{2}$ and $\xi_{3}$, there are two distinct conditions imposed on $\Delta \phi$. These conditions are solved by setting

$$
\xi_{1}=\frac{\xi_{4}^{2}-\xi_{2} \xi_{3}}{2 \xi_{4}-\xi_{2}-\xi_{3}}
$$

implying that $\xi_{3}<\xi_{1}<\xi_{4}$. Equation (2.5) can be viewed as the tuning of the angular momentum to uphold the ring.

The limit $x, y \rightarrow \xi_{2}$ is asymptotic infinity and it can be shown that the solution is asymptotically flat: rescale $\psi$ and $\phi$ by taking $\tilde{\psi}=2 \pi \psi / \Delta \psi$ and $\tilde{\phi}=2 \pi \phi / \Delta \phi$, so that $\Delta \tilde{\phi}=\Delta \tilde{\psi}=2 \pi$, and define $\tilde{A}=A \sqrt{G^{\prime}\left(\xi_{2}\right)} /\left(2 F\left(\xi_{2}\right)\right)$. Via the coordinate transformation

$$
\zeta=\frac{\sqrt{\xi_{2}-y}}{\tilde{A}(x-y)} \quad \text { and } \quad \eta=\frac{\sqrt{x-\xi_{2}}}{\tilde{A}(x-y)},
$$

the asymptotic metric can be written as

$$
d s_{\text {asymp }}^{2}=-d t^{2}+d \zeta^{2}+\zeta^{2} d \tilde{\psi}^{2}+d \eta^{2}+\eta^{2} d \tilde{\phi}^{2} .
$$

The Killing vector $\partial / \partial t$ vanishes when $y \rightarrow-\infty$, and since the metric is regular here the coordinate $Y=-1 / y$ can naturally be extended past $Y=0$ allowing $Y$ to take negative values. The coordinates break down at $y=\xi_{4}$, where $g_{y y}$ blows up. However, this is just a coordinate singularity that can be removed by a change of coordinates. In fact, one finds that there is an $S^{2} \times S^{1}$ horizon at $y=\xi_{4}$ and behind it an $S^{1}$ curvature singularity is hiding at $y=\xi_{1}$. The region $y>\xi_{4}$ is the ergoregion. The ergosurface at $Y=0$ is regular and has topology $S^{2} \times S^{1}$.

Locally, the rotating black ring is expected to look like a boosted black string, and indeed the near singularity behavior of the boosted black string matches that of the black ring (up to numerical factors and distortion, see also sections 4.2,4.4).

The physical quantities such as the ADM mass, the angular momentum, and the surface gravity are given for the black ring in [1. Dimensionless quantities can be formed by multiplying the physical quantities by suitable powers of the mass. For the angular momentum $J$ and the horizon area $\mathcal{A}$ we have

$$
\begin{aligned}
\frac{J^{2}}{M^{3}} & =\frac{32}{27 \pi} \frac{\left(\xi_{4}-\xi_{2}\right)^{3}}{\left(\xi_{3}-\xi_{2}\right)\left(2 \xi_{4}-\xi_{2}-\xi_{3}\right)^{2}}, \\
\frac{\mathcal{A}^{2}}{M^{3}} & =\frac{2048 \pi}{27} \frac{\left(\xi_{3}-\xi_{2}\right)\left(\xi_{4}-\xi_{3}\right)}{\left(2 \xi_{4}-\xi_{2}-\xi_{3}\right)^{2}} .
\end{aligned}
$$

We have used (2.5) to eliminate $\xi_{1}$ from these expressions, and we set $G=c=1$ throughout the paper. In section 4, we compute the physical quantities for the charged 


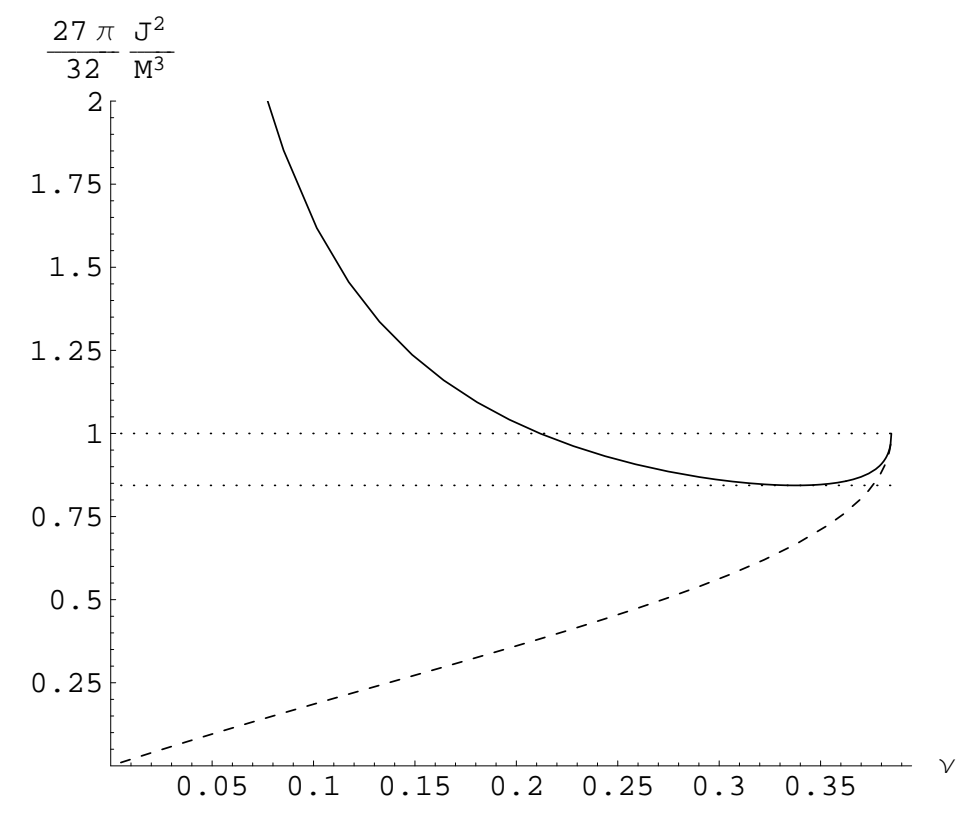

Figure 1: As in Ref. 11, we plot the dimensionless ratio $\frac{27 \pi}{32} \frac{J^{2}}{M^{3}}$ versus $\nu$ for $0<\nu<$ $2 /(3 \sqrt{3})$. The solid line is the ratio for the black ring and the dashed line is the ratio for a Myers-Perry black hole with only one nonzero angular momentum. The dotted lines represent the constant functions 1 and $27 / 32$. For the black ring, $\frac{27 \pi}{32} \frac{J^{2}}{M^{3}}=1$ at $\nu \approx 0.211645$.

black ring, and the mass and angular momentum for the neutral black ring can then be obtained by taking the transformation parameter $\beta$ to zero (see eqs. (4.9) and (4.10) ).

\subsection{An Exact Result for the Black Ring}

Plotting the dimensionless ratio of angular momentum to mass as function of $\nu$, Emparan and Reall found that $J^{2} / M^{3}$ has a global minimum,

$$
\frac{J^{2}}{M^{3}} \geq k \frac{32}{27 \pi}
$$

and they estimated $k \approx 0.8437$ [1]. We reproduce the plot in figure 1 In this section we determine the exact value of $k$ to be $k=27 / 32$. The black ring with $J^{2} / M^{3}=1 / \pi$ is the solution that maximizes the dimensionless measure of entropy, $\mathcal{A} / M^{3 / 2}$.

The quantities $J^{2} / M^{3}$ and $\mathcal{A}^{2} / M^{3}$ are given in (2.8) and (2.9) in terms of the roots, $\xi_{2}, \xi_{3}$, and $\xi_{4}$, of the cubic equation

$$
\nu \xi^{3}-\xi^{2}+1=0
$$


We assume that $0<\nu<2 /(3 \sqrt{3})$ in order for the equation to have three distinct real roots. For $\nu=2 /(3 \sqrt{3})$, the roots $\xi_{3}$ and $\xi_{4}$ coincide. Using standard methods for obtaining the roots of a cubic equation, we find

$$
\begin{aligned}
& \xi_{2}=\frac{1}{3 \nu}\left(1-\cos \frac{\theta}{3}-\sqrt{3} \sin \frac{\theta}{3}\right), \\
& \xi_{3}=\frac{1}{3 \nu}\left(1-\cos \frac{\theta}{3}+\sqrt{3} \sin \frac{\theta}{3}\right), \\
& \xi_{4}=\frac{1}{3 \nu}\left(1+2 \cos \frac{\theta}{3}\right)
\end{aligned}
$$

where

$$
\theta=\cos ^{-1}\left(1-\frac{27}{2} \nu^{2}\right) .
$$

We can now write the dimensionless ratio of angular momentum to mass in terms of $\theta$ as

$$
\frac{27 \pi}{32} \frac{J^{2}}{M^{3}}=\frac{1}{24}\left[8+6 \sqrt{3} \csc \left(\frac{2 \theta}{3}\right)+\sec ^{2}\left(\frac{\theta}{3}\right)\right] .
$$

The global minimum of $\frac{27 \pi}{32} \frac{J^{2}}{M^{3}}$ for the black ring (see figure 1) is found by extremizing the right hand side of (2.12) with respect to $\theta$, and one finds that $\theta=3 \cos ^{-1}(2 / \sqrt{7})$ corresponds to the minimum in figure 11. Solving for $\nu$ using (2.11) we get

$$
\nu_{0}=\frac{1}{3} \sqrt{\frac{2}{3}+\frac{20}{21 \sqrt{7}}} \approx 0.3377 .
$$

Evaluating (2.12) at $\nu=\nu_{0}$ we find the minimum value $k$ to be

$$
\left.k \equiv \frac{27 \pi}{32} \frac{J^{2}}{M^{3}}\right|_{\nu_{0}}=\frac{27}{32}=0.84375,
$$

giving the simple result

$$
M^{3} \leq \pi J^{2}
$$

for the black ring. It is peculiar that the value $k=27 / 32$ - a number produced by extremizing a function which depends solely of the roots of the cubic equation (2.10) - cancels exactly the factor $32 / 27$ which comes from the normalization of the mass and the angular momentum. It would be interesting to understand if there is any significance to this cancellation.

In terms of $\theta$, the dimensionless ratio of the horizon area and the mass is

$$
\frac{\mathcal{A}^{2}}{M^{3}}=\frac{1024 \pi}{81} \tan \left(\frac{\theta}{3}\right)\left[\sqrt{3}-\tan \left(\frac{\theta}{3}\right)\right] .
$$


In the given range, this function has a global maximum for $\nu=\nu_{0}$ given in (2.13), and we find

$$
\left.\frac{\mathcal{A}^{2}}{M^{3}}\right|_{\nu_{0}}=\frac{256 \pi}{27} .
$$

In conclusion, the black ring with $\nu=\nu_{0}$ is the black ring with minimum angular momentum and maximum entropy for the mass given. As $J$ increases, $\mathcal{A}$ decreases, for fixed mass (see also Fig. 3 of [1]).

We find that the value of $\nu$ for which $\frac{27 \pi}{32} \frac{J^{2}}{M^{3}}=1$ is approximately $\nu \approx 0.211645$ (see figure 11). ${ }^{1}$ One finds that for $\frac{1}{\pi} \leq \frac{J^{2}}{M^{3}} \leq \frac{32}{27 \pi}$ there are three distinct solutions with the same asymptotic values $M$ and $J$ : one is the Myers-Perry spherical black hole with $J_{\phi}=0$, the two others are black ring solutions differentiated, for instance, by their entropies.

\section{Review of Hassan-Sen Transformations}

In the early 90 s it was shown [10, 11, 12, 13, 14] that in any string theory the space of classical solutions that are independent of $d$ of the spacetime coordinates has an $\mathrm{O}(d-1,1) \times \mathrm{O}(d-1,1)$ symmetry (or $\mathrm{O}(d) \times \mathrm{O}(d)$ symmetry if the $d$ dimensions are all spatial), where the first factor acts on the left-movers and the second factor acts on the right-movers.

Hassan and Sen [3] showed that in heterotic string theory the group of transformations can be extended so that the group acting on the right-movers includes a subset of the 16 internal coordinates. If the signature of the $d$ coordinates is Lorentzian and the background gauge fields are neutral under $p$ of the $\mathrm{U}(1)$ generators of the gauge group, the group of transformations is $\mathrm{O}(d-1,1) \otimes \mathrm{O}(d+p-1,1)$. These transformations can be used to generate new inequivalent classical solutions from known classical solutions.

The symmetry can be realized explicitly for the low energy effective action, but is valid to all orders in $\alpha^{\prime}$. Hassan and Sen [3] applied the transformations to a magnetic 6-brane solution in ten dimensions to generate a new solution of heterotic supergravity carrying independent electric and magnetic charges as well as antisymmetric tensor field charge. Also, starting from the neutral Kerr black hole in four dimensions, Sen found a charged rotating black hole solution with nontrivial dilaton, magnetic fields, and antisymmetric tensor field [16]. Many other solutions have been generated using these transformations.

We shall be interested in classical solutions in $D$ spacetime dimensions, hence $10-D$ of the ten dimensions for the heterotic string have been compactified; massless

\footnotetext{
${ }^{1}$ The value for $\nu$ given in [1] had a minor typo.
} 
excitations from the compactification and higher derivative terms are not included in the effective action, which is given by

$$
S=\int d^{D} x \sqrt{-G} e^{-\Phi}\left(R^{(D)}+\nabla_{\mu} \Phi \nabla^{\mu} \Phi-\frac{1}{12} H_{\mu \nu \rho} H^{\mu \nu \rho}-\frac{1}{8} F_{\mu \nu} F^{\mu \nu}\right) .
$$

We consider only $\mathrm{U}(1)$ gauge fields, and just a single $\mathrm{U}(1)$ component of the gauge fields has been included in the action (3.1). The antisymmetric 3-form field $H$ includes the U(1) Chern-Simons term,

$$
H_{\mu \nu \rho}=\left(\partial_{\mu} B_{\nu \rho}+\text { cyclic permutations }\right)-\frac{1}{4}\left(A_{\mu} F_{\nu \rho}+\text { cyclic permutations }\right) \text {. }
$$

Throughout the metric $G_{\mu \nu}$ refers to the string frame metric. In $D$ dimensions the Einstein metric is related to the string metric by $G_{\mu \nu}^{\mathrm{E}}=e^{-\frac{2}{D-2} \Phi} G_{\mu \nu}$.

We apply the Hassan-Sen transformations to classical solutions that are independent of the time-direction $x^{0}$ and (at least) one spatial direction $x^{1}$. The transformations of interest to us involve only the $\left(x^{0}, x^{1}\right)$-part of the metric, which we denote by $\hat{G}_{a b}, a, b=0,1$, the 01-part of the antisymmetric tensor $\hat{B}_{a b}$ and the gauge fields $\hat{A}_{a}$. Given such a solution $\left(G_{\mu \nu}, \Phi, A_{\mu}, B_{\mu \nu}\right)$, where $G$ and $B$ are block diagonal, ie. $G_{a i}=B_{a i}=0$ for all $i \neq 0,1$, the transformed solution $\left(\bar{G}_{\mu \nu}, \bar{\Phi}, \bar{A}_{\mu}, \bar{B}_{\mu \nu}\right)$ is computed as follows. Define a $2 \times 2$ matrix $K$ as

$$
K_{a b}=-\hat{B}_{a b}-\hat{G}_{a b}-\frac{1}{4} \hat{A}_{a} \hat{A}_{b}
$$

and a $5 \times 5$ matrix $M$,

$$
M=\left(\begin{array}{ccc}
\left(K^{T}-\eta\right) \hat{G}^{-1}(K-\eta) & \left(K^{T}-\eta\right) \hat{G}^{-1}(K+\eta) & -\left(K^{T}-\eta\right) \hat{G}^{-1} \hat{A} \\
\left(K^{T}+\eta\right) \hat{G}^{-1}(K-\eta) & \left(K^{T}+\eta\right) \hat{G}^{-1}(K+\eta) & -\left(K^{T}+\eta\right) \hat{G}^{-1} \hat{A} \\
-\hat{A}^{T} \hat{G}^{-1}(K-\eta) & -\hat{A}^{T} \hat{G}^{-1}(K+\eta) & \hat{A}^{T} \hat{G}^{-1} \hat{A}
\end{array}\right)
$$

where $\eta=\operatorname{diag}(-1,1)$. In addition to the above assumptions we assume for convenience that $\hat{A}=0$ for the original solution. We have $d=2$ and $p=1$ and the symmetry on the space of solutions is $\mathrm{O}(1,1) \otimes \mathrm{O}(2,1)$. Writing $\Omega \in \mathrm{O}(1,1) \otimes \mathrm{O}(2,1)$ as

$$
\Omega=\left(\begin{array}{ll}
S & 0 \\
0 & R
\end{array}\right)
$$

we can choose $R$ to be on the form

$$
R=\left(\begin{array}{ccc}
\cosh \alpha_{2} & \sinh \alpha_{2} & 0 \\
\sinh \alpha_{2} & \cosh \alpha_{2} & 0 \\
0 & 0 & 1
\end{array}\right)\left(\begin{array}{ccc}
\cosh \alpha_{1} & 0 & \sinh \alpha_{1} \\
0 & 1 & 0 \\
\sinh \alpha_{1} & 0 & \cosh \alpha_{1}
\end{array}\right)
$$


where $\alpha_{1}$ parametrizes boosts that mixes the 0 -direction with the internal coordinate, and $\alpha_{2}$ parametrizes boosts in 01-space. The $\mathrm{O}(1,1)$-transformations are Lorentz boosts of the solution in the 01-plane and we choose $S$ to be the identity matrix.

The Hassan-Sen transformation acts on the solution to give

$$
\begin{aligned}
\bar{M} & =\Omega M \Omega^{T} \\
\bar{\Phi} & =\Phi+\ln \sqrt{\operatorname{det} \bar{G} / \operatorname{det} G}
\end{aligned}
$$

The $\left(x^{0}, x^{1}\right)$-components of the new metric and the fields, $\bar{A}_{a}$ and $\bar{B}_{01}$, are given implicitly by $\bar{M}$ and can be extracted using (3.3) and (3.4) with $G, K$, and $A$ replaced by $\bar{G}, \bar{K}$, and $\bar{A}$. All other field components are unchanged by the transformation. In appendix A.1 and appendix A.2 we give the explicit transformed solution in terms of the original solutions.

\section{Remark: Hassan-Sen transformations with $\alpha_{1}=0$}

Let $(G, \Phi, B=0)$ be a static solution satisfying the above assumptions. It is wellknown [17] that when applying a Lorentz boost with parameter $\alpha(d t \rightarrow d t \cosh \alpha+$ $d z \sinh \alpha$ and $d z \rightarrow d t \sinh \alpha+d z \cosh \alpha)$ and then T-dualizing in the 1-direction, one obtains a new solution $\left(G^{\prime}, \Phi^{\prime}, B^{\prime}\right)$ where the linear momentum created by the boost is exchanged for an $H$-charge. ${ }^{2}$ Boosting the solution $\left(G^{\prime}, \Phi^{\prime}, B^{\prime}\right)$ in 01-space with the same parameter $\alpha$ gives a new solution $\left(G^{\prime \prime}, \Phi^{\prime \prime}, B^{\prime \prime}\right)$ and then T-dualizing again, we find that the resulting solution is exactly the Hassan-Sen transformed solution $(\bar{G}, \bar{\Phi}, \bar{B})$ with $\alpha_{1}=0$ and $\alpha_{2}=2 \alpha$. This also holds true if the original metric has $G_{01} \neq 0$.

If $G_{11}=1$ identically for the original static solution $(G, \Phi, B=0)$ then the last T-duality transformation has no effect: for $G_{11}=1$ the solution $\left(G^{\prime \prime}, \Phi^{\prime \prime}, B^{\prime \prime}\right)=$ $(\bar{G}, \bar{\Phi}, \bar{B})_{\alpha_{1}=0}$ is invariant under T-duality. In section 5 we give an example of a self-T-dual charged black string.

\section{The Charged Black Ring}

\subsection{The Solution}

The rotating black ring solution (2.1) has three Killing vectors corresponding to the coordinates $t, \psi$, and $\phi$. We apply the Hassan-Sen transformation with $\beta=\alpha_{1} / 2$ and $\alpha_{2}=0$ to the $(t, \psi)$-part of the black ring solution (2.1) to find a solution of the theory (3.1) with $D=5$. The transformation is given explicitly in appendix A.2. The

\footnotetext{
${ }^{2}$ The fields are required to fall off appropriately at infinity.
} 
transformed solution is

$$
\begin{aligned}
d s^{2}=-\frac{F(x)}{F(y) h(x, y)^{2}}(d t+ & \sqrt{\left.\frac{\nu}{\xi_{1}} \frac{\xi_{2}-y}{A} \cosh ^{2} \beta d \psi\right)^{2}} \\
+\frac{1}{A^{2}(x-y)^{2}}[ & -F(x)\left(G(y) d \psi^{2}+\frac{F(y)}{G(y)} d y^{2}\right) \\
& \left.+F(y)^{2}\left(\frac{d x^{2}}{G(x)}+\frac{G(x)}{F(x)} d \phi^{2}\right)\right]
\end{aligned}
$$

with fields

$$
\begin{aligned}
e^{-\Phi} & =h(x, y), & B_{t \psi} & =\sinh ^{2} \beta \sqrt{\frac{\nu}{\xi_{1}}} \frac{F(x)\left(\xi_{2}-y\right)}{A F(y) h(x, y)}, \\
A_{t} & =\frac{(x-y) \sinh 2 \beta}{\xi_{1} F(y) h(x, y)}, & A_{\psi} & =-\sinh 2 \beta \sqrt{\frac{\nu}{\xi_{1}}} \frac{F(x)\left(\xi_{2}-y\right)}{A F(y) h(x, y)},
\end{aligned}
$$

The functions $F$ and $G$ are given in (2.2) and

$$
h(x, y)=1+\frac{x-y}{\xi_{1} F(y)} \sinh ^{2} \beta .
$$

The analysis of the metric with respect to signature and regularity works out exactly as for the neutral case. The coordinates $x$ and $y$ are restricted to the regions (2.3) and the coordinates $\psi$ and $\phi$ are periodic with the periods given in (2.4). We note that for $x$ and $y$ in the coordinate regions (2.3), the function $h$ in equation (4.8) is strictly positive.

The asymptotic region is at $x, y \rightarrow \xi_{2}$. Since $h \rightarrow 1$ at infinity, the coordinate transformation (2.6) takes the asymptotic metric to the form (2.7) after the appropriate rescalings. Thus the transformed metric is asymptotically flat.

For $\xi_{1}=\xi_{3}$, the coordinate transformation given in [1] takes the solution given by (4.6) and (4.7) to the solution obtained by applying the Hassan-Sen transformation of section A.2 to the five dimensional Myers-Perry black hole with only one nonzero rotation parameter. This solution is the five dimensional analog of the charged rotating black hole in four dimensions found by Sen [16. It can be generalized to a charged solution with two independent angular momenta by applying a Hassan-Sen transformation to the general five dimensional Myers-Perry rotating black hole.

In the following we assume that $\xi_{1}>\xi_{3}$. Just as in the case of the neutral black ring, regularity requires $\xi_{1}$ to be given by (2.5).

The transformed solution is regular at $y \rightarrow-\infty$, so defining $Y=-1 / y$ we can extend the coordinate region to include $Y<0$ just as for the neutral black ring. At $y=\xi_{4}$, the metric component $g_{y y}$ blows up while the fields stay finite. By a slight 
modification of the coordinate transformation $(t, \psi) \rightarrow(v, \chi)$ given in [1] we obtain new coordinates for which the metric is regular at $y=\xi_{4}$. The new coordinates valid for $y>\xi_{1}$ - are defined as

$$
\begin{aligned}
& d \chi=d \psi+\frac{\sqrt{-F(y)}}{G(y)} d y \\
& d t=d v+\sqrt{\frac{\nu}{\xi_{1}}} \frac{\left(y-\xi_{2}\right) \sqrt{-F(y)}}{A G(y)} \cosh ^{2} \beta d y,
\end{aligned}
$$

so the $t, \psi, y$-part of the metric becomes

$$
\begin{aligned}
d s_{v \chi y}^{2}=-\frac{F(x)}{F(y) h(x, y)^{2}} & \left(d v+\sqrt{\frac{\nu}{\xi_{1}}} \frac{\xi_{2}-y}{A} \cosh ^{2} \beta d \chi\right)^{2} \\
& +\frac{F(x)}{A^{2}(x-y)^{2}}\left[-G(y) d \chi^{2}+2 \sqrt{-F(y)} d \chi d y\right] .
\end{aligned}
$$

The Killing vector $(\partial / \partial t)$ vanishes at $Y=0$, so the region $y>\xi_{4}$ is the ergoregion. The determinant $g_{t t} g_{\psi \psi}-g_{t \psi}^{2}$ has a zero at $y=\xi_{4}$ and since we know that the metric is regular here, the constant- $(v, y)$ surface at $y=\xi_{4}$ defines the event horizon. There is no inner horizon. Both the ergosurface (defined as the constant- $(t, Y)$ surface at $Y=0$ ) and the horizon are topologically $S^{2} \times S^{1}$. The curvature blows up at $y=\xi_{1}$, and the dilaton is singular there; this corresponds to a spacelike $S^{1}$ curvature singularity in the metric.

\subsection{Physical Properties}

Going to the Einstein metric, $G_{\mu \nu}^{\mathrm{E}}=e^{-\frac{2}{3} \Phi} G_{\mu \nu}$, and using the next to leading order behavior of the asymptotic metric we compute the ADM mass and the angular momentum [2]

$$
\begin{aligned}
M & =\frac{3 \pi}{2 A^{2}} \frac{\left(1+\frac{4}{3} \sinh ^{2} \beta\right)\left(\xi_{1}-\xi_{2}\right)}{\xi_{1}^{2} \nu\left(\xi_{3}-\xi_{2}\right)\left(\xi_{4}-\xi_{2}\right)}, \\
J & =\frac{2 \pi}{A^{3}} \frac{\left(\xi_{1}-\xi_{2}\right)^{5 / 2} \cosh ^{2} \beta}{\xi_{1}^{3} \nu^{3 / 2}\left(\xi_{3}-\xi_{2}\right)^{2}\left(\xi_{4}-\xi_{2}\right)^{2}},
\end{aligned}
$$

which reduce to the values for the neutral ring for $\beta \rightarrow 0$. Also, the black ring has an asymptotic electric U(1) charge given by (charges are normalized as in [1] )

$$
Q=\frac{1}{16 \pi} \int_{S^{3} \text { at } \infty} e^{-\Phi} \star F=\frac{\pi\left(\xi_{1}-\xi_{2}\right) \sinh 2 \beta}{A^{2} \xi_{1}^{2} \nu\left(\xi_{3}-\xi_{2}\right)\left(\xi_{4}-\xi_{2}\right)} .
$$


The dimensionless ratio of angular momentum and mass is given by

$$
\frac{J^{2}}{M^{3}}=\frac{\cosh ^{4} \beta}{\left(1+\frac{4}{3} \sinh ^{2} \beta\right)^{3}}\left[\frac{J^{2}}{M^{3}}\right]_{\beta=0} \geq \frac{1}{\pi} \frac{\cosh ^{4} \beta}{\left(1+\frac{4}{3} \sinh ^{2} \beta\right)^{3}},
$$

where we have used the result (2.14) for lower bound on $J^{2} / M^{3}$ for the neutral black ring, and the dimensionless ratio of charge to mass is

$$
\frac{Q}{M}=\frac{2 \sinh 2 \beta}{3\left(1+\frac{4}{3} \sinh ^{2} \beta\right)} .
$$

We note that by taking $\beta$ large we can make $J^{2} / M^{3}$ arbitrarily small while $Q / M$ approaches a constant. Thus the charge helps holding up the black ring allowing us to make the angular momentum arbitrarily small. This was of course not possible for the neutral black ring.

Surprisingly, the ratio $Q / M$ is independent of $\nu$. In fact, we notice that the right hand side of (4.12) is always less than 1 , so that for all $\beta$ we have

$$
|Q| \leq M
$$

with equality in the limit $\beta \rightarrow \infty$. Contrary to other solutions with angular momentum and charge, this bound does not involve the angular momentum.

As a one dimensional object in a five dimensional asymptotically flat spacetime, the black ring can carry local — but not global - fundamental string charge associated with the 3 -form field $H$. Using (3.2) we find that $H$ has only one nonzero component,

$$
H_{t \psi y}=-\frac{\sqrt{\frac{\nu}{\xi_{1}}}\left(\xi_{1}-x\right)^{2} \sinh ^{2} \beta}{A\left[\xi_{1}+x \sinh ^{2} \beta-y \cosh ^{2} \beta\right]^{2}},
$$

and it gives rise to the local fundamental string charge

$$
q_{H}=\frac{1}{16 \pi} \int e^{-\Phi} \star H=\frac{\sinh ^{2} \beta\left(\xi_{1}-\xi_{2}\right)^{1 / 2}}{4 A \xi_{1} \nu^{1 / 2}\left(\xi_{4}-\xi_{2}\right)},
$$

where the integral is over a two sphere parametrized by $x$ and $\phi$ at a constant $\psi$-cut around of the ring. In the limit $\beta \rightarrow \infty$, the dimensionless ratio $q_{H}^{2} / M$ diverges.

From the leading order behavior of the field $A_{\tilde{\psi}}=A_{\psi} \Delta \psi /(2 \pi)$ at infinity we find the magnetic moment $\mu$ of the black ring. In spherical coordinates with radial coordinate $\rho$ and a polar coordinate $\theta$ we have for large $\rho$

$$
A_{\tilde{\psi}}=-\frac{\bar{\mu} \sin ^{2} \theta}{\rho^{2}}
$$


In analog to the normalization of the charges, we normalize the magnetic moment as $\mu=\bar{\mu} A_{3} /(16 \pi)$ where $A_{3}$ is the area of a unit three sphere. We find

$$
\mu=\frac{\pi\left(\xi_{1}-\xi_{2}\right)^{5 / 2} \sinh 2 \beta}{A^{3} \xi_{1}^{3} \nu^{3 / 2}\left(\xi_{3}-\xi_{2}\right)^{2}\left(\xi_{4}-\xi_{2}\right)^{2}} .
$$

The $\nu$ dependence cancels in $\mu / J$ so that the ratio depends only on $\beta$. The gyromagnetic ratio $g$ is defined as $g=2 \mu M /(Q J)$ and we find

$$
g=\frac{3\left(1+\frac{4}{3} \sinh ^{2} \beta\right)}{2 \cosh ^{2} \beta},
$$

so that $g$ is independent of $\nu$. We see from (4.14) that for the charged black ring, the $g$-factor can take values between $3 / 2$ and 2 . The same bounds have been found on the gyromagnetic ratio for the string theory solution describing a dilatonic rotating charged black hole in a four dimensional asymptotically flat spacetime [15]. It should however be noted that there is an ambiguity in the normalization of the magnetic moment; changing the normalization of $\mu$ changes the $g$-factor.

The area of the event horizon is

$$
\mathcal{A}=\frac{16 \pi^{2}}{A^{3}} \frac{\left(\xi_{1}-\xi_{2}\right)\left(\xi_{4}-\xi_{1}\right)^{3 / 2} \cosh ^{2} \beta}{\nu^{3 / 2} \xi_{1}^{3}\left(\xi_{3}-\xi_{2}\right)\left(\xi_{4}-\xi_{2}\right)^{2}\left(\xi_{4}-\xi_{3}\right)} .
$$

As a function of $\beta$, the dimensionless ratio $\mathcal{A} / M^{3 / 2}$ is maximized for $\beta=0$ : for a given mass the neutral black ring always has higher entropy than the charged black ring, and increasing the charge while keeping the mass fixed, the horizon area decreases. This is qualitatively the same behavior as for a charged spherical black hole.

Associated with the horizon is a Killing vector field

$$
\frac{\partial}{\partial v}+\frac{A \xi_{1}^{1 / 2}}{\nu^{1 / 2}\left(\xi_{4}-\xi_{2}\right) \cosh ^{2} \beta} \frac{\partial}{\partial \chi} .
$$

Outside the horizon where the original coordinates are valid, the Killing vector field is given by

$$
\frac{\partial}{\partial t}+\frac{A \nu^{1 / 2} \xi_{1}\left(\xi_{3}-\xi_{2}\right)}{2\left(\xi_{1}-\xi_{2}\right)^{1 / 2} \cosh ^{2} \beta} \frac{\partial}{\partial \tilde{\psi}}
$$

Using the Killing field (4.15) we compute the surface gravity

$$
\kappa=\frac{A\left(\xi_{4}-\xi_{3}\right) \nu^{1 / 2} \xi_{1}}{2\left(\xi_{4}-\xi_{1}\right)^{1 / 2} \cosh ^{2} \beta}
$$

Near the singularity, $y=\xi_{1}+\epsilon$ for small $\epsilon>0$, the metric takes the form

$$
d s^{2} \sim+\epsilon(d v-d \chi)^{2}+d \chi^{2}+\sqrt{\epsilon} d \chi d \epsilon+\epsilon^{2} d \Omega_{2}^{\prime 2},
$$


where we have ignored numerical constants and $x$-dependence (for example, the $(x, \phi)$ part of the metric is only topologically a two sphere). For the fields we find near the singularity

$$
e^{-\Phi} \sim O\left(\epsilon^{-1}\right) \quad \text { and } \quad B_{t z}, A_{t}, A_{z} \sim O(1)
$$

We shall compare this behavior with the near singularity behavior of the Hassan-Sen transformed boosted black string (see section 4.4).

\subsection{Extremal Limit}

In the limit $\nu \rightarrow 0$ the $\xi_{i}$ 's behave as

$$
\xi_{1} \sim \frac{1}{2 \nu}+\frac{\nu}{4}, \quad \xi_{2} \sim-1+\frac{\nu}{2}, \quad \xi_{3} \sim 1+\frac{\nu}{2}, \quad \xi_{4} \sim \frac{1}{\nu}-\nu,
$$

The ratio $J^{2} / M^{3}$ approaches zero when $\beta \rightarrow \infty$, but it diverges for $\nu \rightarrow 0$ (see figure (1). We find an extremal limit of the charged black ring by taking the limit $\beta \rightarrow \infty$ keeping $\lambda \equiv \nu e^{2 \beta}$ fixed. The extremal metric is

$$
\begin{aligned}
d s^{2}=-\frac{1}{\left[1+\frac{\lambda}{2}(x-y)\right]^{2}}\left(d t-\frac{\lambda(1+y)}{2 \sqrt{2} A} d \psi\right)^{2} & \\
& +\frac{1}{A^{2}(x-y)^{2}}\left[\left(y^{2}-1\right) d \psi^{2}+\frac{d y^{2}}{y^{2}-1}+\frac{d x^{2}}{1-x^{2}}+\left(1-x^{2}\right) d \phi^{2}\right]
\end{aligned}
$$

and the fields are

$$
\begin{aligned}
e^{-\Phi} & =1+\frac{\lambda}{2}(x-y), & B_{t \psi} & =-\frac{\lambda(1+y)}{2 \sqrt{2} A\left[1+\frac{\lambda}{2}(x-y)\right]}, \\
A_{t} & =\frac{\lambda(x-y)}{\left[1+\frac{\lambda}{2}(x-y)\right]}, & A_{\psi} & =\frac{\lambda(1+y)}{\sqrt{2} A\left[1+\frac{\lambda}{2}(x-y)\right]} .
\end{aligned}
$$

We now have $-1<x<1$ and $y<-1$, and the periodicities are $\Delta \psi=\Delta \phi=2 \pi$. The solution is asymptotically flat. The curvature blows up at $y \rightarrow-\infty$ and this is a null singularity coinciding with the horizon.

The physical quantities for the extremal solution are

$$
\begin{aligned}
& M=\frac{\pi \lambda}{2 A^{2}}, \quad J=\frac{\pi \lambda}{2^{5 / 2} A^{3}}, \quad Q=\frac{\pi \lambda}{2 A^{2}}, \\
& q_{H}=\frac{\lambda}{8 \sqrt{2} A}, \quad \mu=\frac{\pi \lambda}{2^{5 / 2} A^{3}}, \quad g=2 .
\end{aligned}
$$

Note that the inequality (4.13) is saturated in the extremal limit so that $|Q|=M$. Also, for the extremal ring $\mu=J$ and the $g$-factor is 2. By taking $\lambda$ large we can 
make $J^{2} / M^{3}$ arbitrarily small. The horizon area shrinks to zero, however taking the limit of the surface gravity (4.16) gives a constant, $\kappa \rightarrow \frac{\sqrt{2} A}{\lambda}$. This is similar to the behavior found in [15, 18] for slowly rotating and non-rotating dilatonic charged spherical black hole solutions in string theory.

Defining $r=-1 / y$ and considering small $r>0$ we find that the near horizon/singularity behavior is

$$
d s^{2} \sim-\frac{4 r^{2}}{\lambda^{2}}\left(d t+\frac{\lambda}{2 \sqrt{2} A r} d \psi\right)^{2}+\frac{1}{A^{2}}\left(d \psi^{2}+d r^{2}+r^{2} d \Omega_{2}^{2}\right)
$$

with

$$
e^{-\Phi} \sim \frac{\lambda}{2 r}, \quad A_{t}, A_{z}, B_{t z} \rightarrow \text { constant }
$$

We compare this with the near-singularity behavior of the extremal limit of the charged boosted black string in section 4.4.

\subsection{A Charged Boosted Black String}

The local behavior of the neutral rotating black ring is like that of a boosted black string, hence we expect that the charged black ring behaves locally as a boosted black string with similar charges and fields. We check this by comparing the near singularity behavior of the charged black ring to that of a charged black string obtained from the boosted black string by the Hassan-Sen transformation of appendix A.2.

The black string metric in five dimensions is the four dimensional Schwarzschild solution times $\mathbb{R}$,

$$
d s^{2}=-\left(1-\frac{r_{0}}{r}\right) d t^{2}+d z^{2}+\left(1-\frac{r_{0}}{r}\right)^{-1} d r^{2}+r^{2} d \Omega_{2}^{2} .
$$

Applying a Lorentz boost to the solution (4.17) by taking $d t \rightarrow d t \cosh \alpha+d z \sinh \alpha$ and $d z \rightarrow d t \sinh \alpha+d z \cosh \alpha$, we obtain the metric for the boosted black string

$$
\begin{gathered}
d s^{2}=-\left(1-\frac{r_{0} \cosh ^{2} \alpha}{r}\right) \\
d t^{2}+\frac{r_{0} \sinh 2 \alpha}{r} d t d z+\left(1+\frac{r_{0} \sinh ^{2} \alpha}{r}\right) d z^{2} \\
+\left(1-\frac{r_{0}}{r}\right)^{-1} d r^{2}+r^{2} d \Omega_{2}^{2}
\end{gathered}
$$

T-dualizing the metric (4.18) gives the solution for the non-extremal fundamental black string (see section [5). Now instead apply the Hassan-Sen transformation of 
section A.2 to (4.18). The transformed solution is given by

$$
\begin{gathered}
d s^{2}=-\frac{r\left(r-r_{0} \cosh ^{2} \alpha\right)}{\left[r+r_{0} \cosh ^{2} \alpha \sinh ^{2} \beta\right]^{2}}\left(d t-\frac{r_{0} \sinh 2 \alpha \cosh ^{2} \beta}{2\left(r-r_{0} \cosh ^{2} \alpha\right)} d z\right)^{2} \\
+\frac{r-r_{0}}{r-r_{0} \cosh ^{2} \alpha} d z^{2}+\left(1-\frac{r_{0}}{r}\right)^{-1} d r^{2}+r^{2} d \Omega_{2}^{2}, \\
e^{-\Phi}=1+\frac{r_{0} \cosh ^{2} \alpha \sinh ^{2} \beta}{r}, \quad B_{t z}=-\frac{r_{0} \sinh 2 \alpha \sinh ^{2} \beta}{2\left[r+r_{0} \cosh ^{2} \alpha \sinh ^{2} \beta\right]}, \\
A_{t}=\frac{r_{0} \cosh ^{2} \alpha \sinh 2 \beta}{\left[r+r_{0} \cosh ^{2} \alpha \sinh ^{2} \beta\right]}, \quad A_{z}=\frac{r_{0} \sinh 2 \alpha \sinh _{2} \beta}{2\left[r+r_{0} \cosh ^{2} \alpha \sinh ^{2} \beta\right]} .
\end{gathered}
$$

The solution is regular at $r=r_{0} \cosh ^{2} \alpha$ and the coordinate singularity at $r=r_{0}$ can be removed by a coordinate transformation (valid for $r<r_{0} \cosh ^{2} \alpha$ )

$$
\begin{aligned}
& d v=d t+\frac{\sqrt{r_{0} \cosh ^{2} \alpha / r-1}}{1-r_{0} / r} \frac{r_{0} \sinh 2 \alpha \cosh ^{2} \beta}{2\left(r-r_{0} \cosh ^{2} \alpha\right)} d r \\
& d w=d z+\frac{\sqrt{r_{0} \cosh ^{2} \alpha / r-1}}{1-r_{0} / r} d r .
\end{aligned}
$$

In these coordinates the metric becomes

$$
\begin{gathered}
d s^{2}=-\frac{r\left(r-r_{0} \cosh ^{2} \alpha\right)}{\left[r+r_{0} \cosh ^{2} \alpha \sinh ^{2} \beta\right]^{2}}\left(d v-\frac{r_{0} \sinh 2 \alpha \cosh ^{2} \beta}{2\left(r-r_{0} \cosh ^{2} \alpha\right)} d w\right)^{2} \\
\quad+\frac{r-r_{0}}{r-r_{0} \cosh ^{2} \alpha} d w^{2}+2\left(r_{0} \cosh ^{2} \alpha / r-1\right)^{-1 / 2} d r d w+r^{2} d \Omega_{2}^{2},
\end{gathered}
$$

and it is regular at $r=r_{0}$. The Killing vector $\partial / \partial t$ becomes null at $r=r_{0} \cosh ^{2} \alpha$, and the determinant $g_{t t} g_{z z}-g_{t z}^{2}$ vanishes at $r=r_{0}$; the region $r_{0}<r<r_{0} \cosh ^{2} \alpha$ is the ergoregion for the string and $r=r_{0}$ defines the horizon. There is a curvature singularity at $r=0$.

The physical quantities can be calculated as in the previous section. We find (the linear momentum is computed following [17])

$$
\begin{aligned}
m & =\frac{r_{0}}{4}\left[1+\cosh ^{2} \alpha \cosh 2 \beta\right], & P_{z} & =\frac{r_{0}}{8} \sinh 2 \alpha \cosh ^{2} \beta, \\
q & =\frac{r_{0}}{4} \cosh ^{2} \alpha \sinh 2 \beta, & Q_{H} & =-\frac{r_{0}}{8} \sinh 2 \alpha \sinh ^{2} \beta
\end{aligned}
$$

and the surface gravity is

$$
\kappa=\frac{1}{2 r_{0} \cosh \alpha \cosh ^{2} \beta} .
$$


The near singularity behavior of the solution is (ignoring numerical constants)

$$
\begin{array}{r}
d s^{2} \sim+r(d v+d w)^{2}+d w^{2}-\sqrt{r} d r d w+r^{2} d \Omega_{2}^{2}, \\
e^{-\Phi} \sim O\left(r^{-1}\right), \quad B_{t z}, A_{t}, A_{z} \sim \text { constants }
\end{array}
$$

Qualitatively, this agrees with the near singularity behavior of the charged black ring. For the black ring, the two sphere is distorted and so is the $S^{1}$ around the ring.

\section{Extremal limit}

There is an extremal limit defined by taking $\beta \rightarrow \infty$ while keeping $\lambda_{0} \equiv \frac{r_{0}}{4} e^{2 \beta}$ fixed. The extremal solution is

$$
\begin{gathered}
d s^{2}=-\frac{1}{\left[1+\lambda_{0} \cosh ^{2} \alpha / r\right]^{2}}\left(d t-\frac{\lambda_{0} \sinh 2 \alpha}{2 r} d z\right)^{2}+d z^{2}+d r^{2}+r^{2} d \Omega_{2}^{2}, \\
e^{-\Phi}=1+\frac{\lambda_{0} \cosh ^{2} \alpha}{r}, \quad B_{t z}=-\frac{\lambda_{0} \sinh 2 \alpha}{2\left[r+\lambda_{0} \cosh ^{2} \alpha\right]}, \\
A_{t}=\frac{2 \lambda_{0} \cosh ^{2} \alpha}{\left[r+\lambda_{0} \cosh ^{2} \alpha\right]}, \quad A_{z}=\frac{\lambda_{0} \sinh 2 \alpha}{\left[r+\lambda_{0} \cosh ^{2} \alpha\right]} .
\end{gathered}
$$

At $r=0$ there is a null singularity coinciding with the horizon. The near singularity behavior for the extremal boosted string is

$$
\begin{gathered}
d s^{2}=-\frac{r^{2}}{\lambda_{0}^{2} \cosh ^{4} \alpha}\left(d t-\frac{\lambda_{0} \sinh 2 \alpha}{2 r} d z\right)^{2}+d z^{2}+d r^{2}+r^{2} d \Omega_{2}^{2}, \\
e^{-\Phi}=\frac{\lambda_{0} \cosh ^{2} \alpha}{r}, \quad B_{t z}, A_{t}, A_{z} \sim \text { constants }
\end{gathered}
$$

As expected, this agrees with the near singularity behavior of the extremal charged black ring.

\section{Black Strings with Charge}

The most general five dimensional black string solution labelled by mass, angular momentum, electric charge per length, and fundamental string charge was analyzed by Mahapatra in [19]. The solution was found by applying a Hassan-Sen transformation with general $\alpha_{1}$ and $\alpha_{2}$ to the rotating black string vacuum solution obtained as the four dimensional Kerr solution times a flat direction. In this section we consider 
a special case where the Hassan-Sen transformation is applied to the neutral nonrotating black string and we find that in one extremal limit this solution describes traveling waves in a fundamental string background.

Applying the Hassan-Sen transformation of section A.1 to the neutral black string (4.17), we find a solution with the metric

$$
d s^{2}=-\frac{4 r\left(r-r_{0}\right)-r_{0}^{2} b^{2}}{4(r+\tilde{r})^{2}} d t^{2}+\frac{r_{0} b}{r+\tilde{r}} d t d z+d z^{2}+\left(1-\frac{r_{0}}{r}\right)^{-1} d r^{2}+r^{2} d \Omega_{2}^{2},
$$

and with fields

$$
e^{-\Phi}=1+\frac{\tilde{r}}{r}, \quad B_{t z}=\frac{r_{0} b}{2(r+\tilde{r})}, \quad A_{t}=\frac{r_{0} a}{r+\tilde{r}}, \quad A_{z}=0 .
$$

We have defined

$$
a=\sinh \alpha_{1}, \quad b=\cosh \alpha_{1} \sinh \alpha_{2}, \quad \tilde{r}=\frac{r_{0}}{2}\left(\cosh \alpha_{1} \cosh \alpha_{2}-1\right) .
$$

There is an $S^{2} \times \mathbb{R}$ horizon at $r=r_{0}$ and the metric (5.19) has a curvature singularity at $r=0$. The fields are regular everywhere, except at $r=0$ where the dilaton blows up. The solution represents a charged black string with mass per length $m$, electric charge per length $q$, fundamental string charge $Q_{H}$, and linear momentum $P_{z}$ along the string, given by

$$
m=\left(r_{0} / 4\right)\left(1+\cosh \alpha_{1} \cosh \alpha_{2}\right), \quad q=r_{0} a / 4, \quad Q_{H}=P_{z}=r_{0} b / 8 .
$$

Expressed in terms of the three independent physical parameters $m, q$, and $Q_{H}$, we have

$$
r_{0}=\frac{2}{m}\left(m^{2}-q^{2}-4 Q_{H}^{2}\right)
$$

so we must require

$$
m^{2} \geq q^{2}+4 Q_{H}^{2}
$$

for the solution not to be naked singular. There are two extremal limits that saturate the inequality (5.21); we study these at the end of this section.

Consider now the solution for a black string with fundamental string charge in five dimensions [21, 17, 20] given by

$$
\begin{aligned}
d s^{2} & =-\frac{r-r_{0}^{\prime}}{r+r_{0}^{\prime} \sinh ^{2} \alpha^{\prime}} d t^{2}+\frac{r}{r+r_{0}^{\prime} \sinh ^{2} \alpha^{\prime}} d z^{2}+\left(1-\frac{r_{0}^{\prime}}{r}\right)^{-1} d r^{2}+r^{2} d \Omega_{2}^{2} \\
e^{-\Phi} & =1+\frac{r_{0}^{\prime} \sinh ^{2} \alpha^{\prime}}{r} \\
B_{t z} & =\frac{r_{0}^{\prime} \sinh 2 \alpha^{\prime}}{2\left(r+r_{0}^{\prime} \sinh ^{2} \alpha^{\prime}\right)},
\end{aligned}
$$


and $A_{t}=A_{z}=0$. In the extremal limit $\alpha^{\prime} \rightarrow \infty$ with $r_{0}^{\prime} e^{2 \alpha^{\prime}}$ constant, this solution describes the fields outside a straight fundamental string [22, 20].

The solution (5.22) can be obtained from the neutral black string (4.17) by first applying a Lorentz boost and then T-dualizing to exchange the linear momentum with $H$-charge [17, 20, 21].

Applying a Lorentz boost with boost parameter $\alpha^{\prime}$ to the solution (15.22) we obtain a solution describing a boosted black string with $H$-charge. As discussed in section 3 this is exactly the same solution as the transformed solution in (5.19) and (5.20) with $\alpha_{1}=0, \alpha_{2}=2 \alpha^{\prime}$, and $r_{0}=r_{0}^{\prime}$, and it is invariant under T-duality.

Returning to the solution given in (5.19) and (5.20) we find that the inequality (5.21) can be saturated by taking $\alpha_{1} \rightarrow \infty$ with $\alpha_{2}$ fixed or vice versa. We consider both cases in the following.

\section{Extremal limit I}

The inequality (5.21) is saturated by taking $\alpha_{2} \rightarrow \infty$ while keeping $\alpha_{1}$ and $R \equiv \frac{1}{4} r_{0} e^{\alpha_{2}}$ constant. In this limit the electric field vanishes, and the $B$-field and the dilaton behave just as the fields for a straight fundamental string [21, 17, 20], but whereas the fundamental string is boost invariant along the $z$-direction, this solution is not.

Introducing null coordinates $u=\frac{1}{\sqrt{2}}(z+t)$ and $v=\frac{1}{\sqrt{2}}(z-t)$ the Einstein metric for this extremal solution can be written

$$
d s_{\text {Einstein }}^{2}=\left(1+\frac{M}{r}\right)^{-1 / 3}\left(2 d u d v+\frac{2 M}{r} d u^{2}\right)+\left(1+\frac{M}{r}\right)^{2 / 3}\left(d r^{2}+r^{2} d \Omega_{2}^{2}\right)
$$

where $M=R \cosh \alpha_{1}$. This matches exactly the traveling wave solutions found by Garfinkle 23. Thus the solution describes constant spherically symmetric traveling waves along a fundamental black string.

\section{Extremal limit II}

Taking $\alpha_{1} \rightarrow \infty$ while keeping $\alpha_{2}$ and $R^{\prime} \equiv \frac{1}{4} r_{0} e^{\alpha_{1}}$ constant gives another extremal solution with charges that saturate the inequality (5.21). This extremal solution is given by the metric

$$
d s^{2}=-\frac{r^{2}-K^{2}}{(r+M)^{2}} d t^{2}+\frac{2 K}{r+M} d t d z+d z^{2}+d r^{2}+r^{2} d \Omega_{2}^{2},
$$

where now $M=R^{\prime} \cosh \alpha_{2}$ and $K=R^{\prime} \sinh \alpha_{2}$, and the fields

$$
e^{-\bar{\Phi}}=1+\frac{M}{r}, \quad B_{t z}=\frac{K}{r+M}, \quad A_{t}=\frac{2 \sqrt{M^{2}-K^{2}}}{r+M}, \quad A_{z}=0 .
$$


The physical mass per length is $m=M / 2$, the electrical charge per length is $q=$ $\frac{1}{2} \sqrt{M^{2}-K^{2}}$, and the fundamental string charge $Q_{h}=K / 4$ equals the linear momentum $P_{z}$ along the string.

\section{Discussion}

We have constructed a charged black ring solution to five dimensional heterotic supergravity and studied its properties. The solution is labelled by mass, angular momentum, and electric charge, and it also carries local fundamental string charge. We found that the lower bound on the dimensionless ratio $J^{2} / M^{3}$ could be arbitrarily small, because the charge would help supporting the ring from collapsing.

As mentioned in section 4.1, the solution given by (4.6) and (4.7) describes a charged rotating black ring for $\xi_{1}>\xi_{3}$ and a charged rotating black hole for $\xi_{1}=$ $\xi_{3}$. The latter can also be obtained by applying the Hassan-Sen transformation of appendix A.2 to the five dimensional Myers-Perry black hole with one of the rotation parameters set to zero. For a given $\beta$, the ratio $|Q| / M$ is fixed, and one can then use the parameter $\nu$ to set the value of $J^{2} / M^{3}$. This holds for both the charged black ring and the charged spherical black hole solutions. The behavior of $J^{2} / M^{3}$ as a function of $\nu$ is as for the neutral black ring (see figure 1), just rescaled by a $\beta$-dependent factor. Hence there exists a range of values of $J^{2} / M^{3}$ such that there are three distinct solutions with the same asymptotic charges: one is the Hassan-Sen transform of the Myers-Perry black hole with just one nonzero angular momentum, and the two others are black ring solutions (one has greater horizon area than the other). This is an extension of the non-uniqueness result of Ref. [1] to charged solutions of low energy heterotic string theory.

The charged black hole found here can be generalized by applying a HassanSen transformation to the general five dimensional Myers-Perry black hole with two independent rotation parameters.

For the charged black ring we found a curious relationship between the mass and the charge: $|Q| \leq M$. The inequality is saturated in the extremal limit, encouraging that the extremal solution may actually be supersymmetric. This requires further investigation.

The stability of the black ring solutions is an open question. The black rings resemble thin black strings, in particular for small $\nu$, so one may expect to find a classical Gregory-Laflamme instability mode 24]. If it exists, such an instability mode would generate ripples in the horizon, and due to the rotation the ripples would radiate away and one would expect the ring to collapse to a rotating black hole. 
Very recently, Hong and Teo [25] presented a new form of the C-metric, and they expressed the neutral black ring metric (2.1) in terms of these new coordinates. Some expressions in this paper simplify when the coordinates of Hong and Teo are used; in particular, the calculation of the exact value of the lower bound of $J^{2} / M^{3}$ simplifies drastically.

\section{Acknowledgement}

It is a great pleasure to thank Gary Horowitz for innumerable helpful discussions and guidance throughout this work, and for comments on the draft. I am grateful to Roberto Emparan and Harvey Reall for comments and also for bringing Ref. 25] to my attention. I would like to thank the Niels Bohr Institute for hospitality during the early stages of the project. This work was supported by the Danish Research Agency and NSF grant PHY-0070895.

\section{A Hassan-Sen Transformed Solutions}

In this appendix we give explicit expressions for solutions obtained by the Hassan-Sen solution generating technique reviewed in section 3,

\section{A.1 Transformation of a Static Metric}

Let $\left(G_{\mu \nu}, \Phi\right)$ be a static solution independent of the time-direction $x^{0}$ and the spatial direction $x^{1}$ with no gauge fields and no antisymmetric tensor fields. The transformations considered here involve only the $\left(x^{0}, x^{1}\right)$-part of the metric. We assume that the metric is block diagonal, ie. $G_{a i}=0$ for all $i \neq 0,1$ and $a, b=0,1$. The transformed solution is then given by the metric

$$
\begin{gathered}
\bar{G}_{00}=\frac{4}{X(G)}\left\{\cosh ^{2} \alpha_{1} \sinh ^{2} \alpha_{2} G_{00}^{-1}\left(1+G_{00}\right)^{2}+G_{11}^{-1}\left[\left(1+G_{11}\right)+\cosh \alpha_{2}\left(1-G_{11}\right)\right]^{2}\right\}, \\
\bar{G}_{01}=\frac{4 \sinh \alpha_{2}}{X(G)}\left\{\cosh \alpha_{1} G_{00}^{-1}\left(1+G_{00}\right)\left[\left(1-G_{00}\right)+\cosh \alpha_{1} \cosh \alpha_{2}\left(1+G_{00}\right)\right]\right. \\
\left.+G_{11}^{-1}\left(1-G_{11}\right)\left[\left(1+G_{11}\right)+\cosh \alpha_{2}\left(1-G_{11}\right)\right]\right\}, \\
\bar{G}_{11}=\frac{4}{X(G)}\left\{G_{00}^{-1}\left[\left(1-G_{00}\right)+\cosh \alpha_{1} \cosh \alpha_{2}\left(1+G_{00}\right)\right]^{2}+\sinh ^{2} \alpha_{2} G_{11}^{-1}\left(1-G_{11}\right)^{2}\right\},
\end{gathered}
$$


where

$$
\begin{aligned}
X(G)=G_{00}^{-1} G_{11}^{-1}\left\{\left(1-G_{00}\right)[\right. & \left.\left(1+G_{11}\right)+\cosh \alpha_{2}\left(1-G_{11}\right)\right] \\
& \left.+\cosh \alpha_{1}\left(1+G_{00}\right)\left[\left(1-G_{11}\right)+\cosh \alpha_{2}\left(1+G_{11}\right)\right]\right\}^{2} ;
\end{aligned}
$$

the dilaton

$$
\bar{\Phi}=\Phi+\ln \sqrt{\frac{16}{G_{00} G_{11} X(G)}} ;
$$

the gauge fields

$$
\begin{aligned}
& \bar{A}_{0}=\frac{2 \sinh \alpha_{1}\left(1+G_{00}\right)\left[\left(1+G_{11}\right)+\cosh \alpha_{2}\left(1-G_{11}\right)\right]}{\sqrt{G_{00} G_{11} X(G)}}, \\
& \bar{A}_{1}=\frac{2 \sinh \alpha_{1} \sinh \alpha_{2}\left(1+G_{00}\right)\left(1-G_{11}\right)}{\sqrt{G_{00} G_{11} X(G)}}
\end{aligned}
$$

and an antisymmetric tensor field

$$
\bar{B}_{01}=\frac{\sinh \alpha_{2}}{\sqrt{G_{00} G_{11} X(G)}}\left[\left(1-G_{00}\right)\left(1-G_{11}\right)+\cosh \alpha_{1}\left(1+G_{00}\right)\left(1+G_{11}\right)\right] .
$$

Even when the original solution is asymptotically flat, the transformed metric is not necessarily so. However, if the original solution is asymptotically flat such that $G_{00} \rightarrow-1$ and $G_{11} \rightarrow 1$ at infinity, then $\bar{G}_{00} \rightarrow-1, \bar{G}_{01} \rightarrow 0$, and $\bar{G}_{11} \rightarrow 1$ at infinity, so the transformed solution is also asymptotically flat.

If $G_{11}=1$ identically, then $\bar{G}_{11}=1$ identically and there will be no magnetic U(1) field, $\bar{A}_{1}=0$. Note that $\mathrm{U}(1)$ gauge fields are generated only if $\alpha_{1} \neq 0$ and that an antisymmetric tensor field is generated only if $\alpha_{2} \neq 0$. Also, $\bar{G}_{01}$ vanishes for $\alpha_{2}=0$.

\section{A.2 Transformation of a Stationary Metric $\left(\alpha_{2}=0\right)$}

Let $\left(G_{\mu \nu}, \Phi\right)$ be a solution satisfying the conditions from section A.1 only that now we allow for an off-diagonal term $G_{01} \neq 0$. Setting $\beta=\alpha_{1} / 2$ and $\alpha_{2}=0$, the transformed 
solution is

$$
\begin{array}{rlrl}
\bar{G}_{00} & =\frac{G_{00}}{\left[1+\left(1+G_{00}\right) \sinh ^{2} \beta\right]^{2}} \\
\bar{G}_{01} & =\frac{G_{01} \cosh ^{2} \beta}{\left[1+\left(1+G_{00}\right) \sinh ^{2} \beta\right]^{2}} \\
\bar{G}_{11} & =\frac{G_{00}^{-1} G_{01}^{2} \cosh ^{4} \beta}{\left[1+\left(1+G_{00}\right) \sinh ^{2} \beta\right]^{2}}+G_{00}^{-1} \operatorname{det} \hat{G}, & & \\
\bar{\Phi} & =\Phi-\ln \left[1+\left(1+G_{00}\right) \sinh ^{2} \beta\right], & \bar{B}_{01} & =-\frac{G_{01} \sinh ^{2} \beta}{1+\left(1+G_{00}\right) \sinh ^{2} \beta} \\
\bar{A}_{0} & =\frac{\left(1+G_{00}\right) \sinh ^{2} \beta}{1+\left(1+G_{00}\right) \sinh ^{2} \beta}, & \bar{A}_{1} & =\frac{G_{01} \sinh _{2} \beta}{1+\left(1+G_{00}\right) \sinh ^{2} \beta} .
\end{array}
$$

For $G_{01}=0$, this is just the $\alpha_{2}=0$ case of the previous section.

If the original solution is asymptotically flat with $G_{00} \rightarrow-1$ and $G_{01} \rightarrow 0$ at infinity, then the new solution is asymptotically flat, and the matter fields approach zero at infinity.

\section{References}

[1] R. Emparan and H. S. Reall, "A rotating black ring in five dimensions," Phys. Rev. Lett. 88, 101101 (2002) arXiv:hep-th/0110260.

[2] R. C. Myers and M. J. Perry, "Black Holes In Higher Dimensional Space-Times," Annals Phys. 172, 304 (1986).

[3] S. F. Hassan and A. Sen, "Twisting classical solutions in heterotic string theory," Nucl. Phys. B 375, 103 (1992) arXiv:hep-th/9109038.

[4] J. P. Gauntlett, J. B. Gutowski, C. M. Hull, S. Pakis and H. S. Reall, "All supersymmetric solutions of minimal supergravity in five dimensions," arXiv:hep-th/0209114.

[5] H. S. Reall, "Higher dimensional black holes and supersymmetry," arXiv:hep-th/0211290

[6] J. C. Breckenridge, R. C. Myers, A. W. Peet and C. Vafa, "D-branes and spinning black holes," Phys. Lett. B 391, 93 (1997) arXiv:hep-th/9602065.

[7] A. Strominger and C. Vafa, "Microscopic Origin of the Bekenstein-Hawking Entropy," Phys. Lett. B 379, 99 (1996) arXiv:hep-th/9601029. 
[8] R. Emparan and H. S. Reall, "Generalized Weyl solutions," Phys. Rev. D 65, 084025 (2002) arXiv:hep-th/0110258.

[9] A. Chamblin and R. Emparan, "Bubbles in Kaluza-Klein theories with space- or time-like internal dimensions," Phys. Rev. D 55, 754 (1997) arXiv:hep-th/9607236.

[10] G. Veneziano, "Scale factor duality for classical and quantum strings," Phys. Lett. B 265, 287 (1991).

[11] K. A. Meissner and G. Veneziano, "Symmetries of cosmological superstring vacua," Phys. Lett. B 267, 33 (1991).

[12] A. Sen, "O(d) x $\mathrm{O}(\mathrm{d})$ symmetry of the space of cosmological solutions in string theory, scale factor duality and two-dimensional black holes," Phys. Lett. B 271, 295 (1991).

[13] A. Sen, "Twisted black p-brane solutions in string theory," Phys. Lett. B 274, 34 (1992) arXiv:hep-th/9108011.

[14] M. Gasperini, J. Maharana and G. Veneziano, "From trivial to nontrivial conformal string backgrounds via $\mathrm{O}(\mathrm{d}, \mathrm{d})$ transformations," Phys. Lett. B 272, 277 (1991).

[15] J. H. Horne and G. T. Horowitz, "Rotating Dilaton Black Holes," Phys. Rev. D 46, 1340 (1992) arXiv:hep-th/9203083.

[16] A. Sen, "Rotating charged black hole solution in heterotic string theory," Phys. Rev. Lett. 69, 1006 (1992) arXiv:hep-th/9204046.

[17] J. H. Horne, G. T. Horowitz and A. R. Steif, "An Equivalence between momentum and charge in string theory," Phys. Rev. Lett. 68, 568 (1992) arXiv:hep-th/9110065.

[18] D. Garfinkle, G. T. Horowitz and A. Strominger, "Charged Black Holes In String Theory," Phys. Rev. D 43, 3140 (1991) [Erratum-ibid. D 45, 3888 (1992)].

[19] S. Mahapatra, "On the rotating charged black string solution," Phys. Rev. D 50, 947 (1994) arXiv:hep-th/9301125.

[20] G. T. Horowitz and A. Strominger, "Black Strings And P-Branes," Nucl. Phys. B 360, 197 (1991).

[21] G. T. Horowitz, "The dark side of string theory: Black holes and black strings," arXiv:hep-th/9210119. 
[22] A. Dabholkar, G. W. Gibbons, J. A. Harvey and F. Ruiz Ruiz, "Superstrings And Solitons," Nucl. Phys. B 340, 33 (1990).

[23] D. Garfinkle, "Black String Traveling Waves," Phys. Rev. D 46, 4286 (1992) arXiv:gr-qc/9209002.

[24] R. Gregory and R. Laflamme, "Black Strings And P-Branes Are Unstable," Phys. Rev. Lett. 70, 2837 (1993) arXiv:hep-th/9301052.

[25] K. Hong and E. Teo, "A new form of the C-metric," Class. Quant. Grav. 20, 3269 (2003) arXiv:gr-qc/0305089. 